\title{
Physical similarity (and not quantity representation) drives perceptual comparison of numbers: Evidence from two Indian notations
}

\author{
Javier García-Orza • Manuel Perea • \\ Reem Abu Mallouh • Manuel Carreiras
}

Published online: 20 January 2012

(C) Psychonomic Society, Inc. 2012

\begin{abstract}
Numerical quantity seems to affect the response in any task that involves numbers, even in tasks that do not demand access to quantity (e.g., perceptual tasks). That is, readers seem to activate quantity representations upon the mere presentation of integers. One important piece of evidence in favor of this view comes from the finding of a distance effect in perceptual tasks: When one compares two numbers, response times (RTs) are a function of the numerical distance between them. However, recent studies have suggested that the physical similarity between Arabic numbers is strongly correlated with their numerical distance, and that the former could be a better predictor of RT data in perceptual tasks in which magnitude processing is not required (Cohen, 2009a). The present study explored the Persian and Arabic versions of Indian numbers (Exps. 1 and 2, respectively). Naïve participants (speakers of Spanish) and users of these notations (Pakistanis and Jordanians) participated in a physical same-different matching task. The RTs of users of the Indian notations were regressed on
\end{abstract}

\section{J. García-Orza $(\bowtie)$}

Departamento de Psicología Básica, Facultad de Psicología,

Universidad de Málaga,

Campus de Teatinos $\mathrm{s} / \mathrm{n}$,

29071 Málaga, Spain

e-mail: jgorza@uma.es

M. Perea

Universitat de València,

Valencia, Spain

R. A. Mallouh $\cdot$ M. Carreiras

Basque Center for Cognition Brain and Language,

Donostia-San Sebastián, Spain

M. Carreiras

IKERBASQUE, Basque Foundation for Science,

Bilbao, Spain perceptual similarity (estimated from the Spanish participants' RTs) and numerical distance. The results showed that, regardless of the degree of correlation between the perceptual similarity function and the numerical distance function, the critical predictor for RTs was perceptual similarity. Thus, participants do not automatically activate Indian integers' quantity representations, at least not when these numbers are presented in simple perceptual tasks.

Keywords Automaticity - Quantity representation . Perceptual similarity Indian numbers

Are semantic (numerical) representations activated upon the presentation of numbers? One common assumption in the literature on numerical cognition is that, when people are presented with Arabic integers, the corresponding semantic representations are automatically activated (Tzelgov, 1997; see Tzelgov \& Ganor-Stern, 2005, for a review). One of the key phenomena supporting this view is the distance effect: When we compare two numbers, the larger the numerical difference between them, the faster the response times (RTs). In an influential experiment, Moyer and Landauer (1967) presented pairs of integers (e.g., 5-7) and asked participants to judge which of the two was larger. They found that RTs decreased as the numerical distance between the two numbers increased, and they argued that this relationship followed the Welford (1960) function (see Eq. 1 below).

$\mathrm{RT}=$ constant $1+$ constant 2 log

[Larger quantity/(Larger quantity-Smaller quantity)].

Of course, when using a numerical task, it is not surprising that number quantities would be activated. The critical 
support for the automaticity of quantity representations comes from experiments on the distance effect in which quantity processing is not part of the task (see Tzelgov, 1997). ${ }^{1}$ In this light, Dehaene and Akhavein (1995) presented participants with pairs of Arabic digits, pairs of verbal numbers, or mixed pairs (one digit and one verbal number) and asked them to decide whether the pair referred to the same quantity (numerical same-different task) or whether they were physically identical (physical same-different task). The numerical distance effect occurred in the numerical task for all conditions; more importantly, this effect also occurred for pairs of the same notation in the physical task. Dehaene and Akhavein concluded that this finding supports the automatic access to quantity, even in perceptual tasks; the lack of effects in the mixed conditions was attributed to the physical differences between the verbal and Arabic numbers. More recently, Ganor-Stern and Tzelgov (2008, Exp. 2) replicated the Dehaene and Akhavein study using pairs of Arabic-Indian (a special notation used in Arabic countries; e.g., ' $, r, r$, and ₹-respectively, 1, 2, 3, and 4) or Arabic and Indian (mixed condition) numbers. The researchers found distance effects in the numerical task, but not in the perceptual task, for any notationallegedly due to a lack of sensitivity of their measures. Importantly, Ganor-Stern and Tzelgov (2008) claimed that evidence of automatic processing of quantity emerged in their experiment, because participants were slower and made more errors in responding "different" for different-notation pairs with the same numerical values (e.g., $2-r$ ), as compared to pairs with different numerical values $(3-r)$.

In a recent study, Cohen (2009a) identified a potential confound in previous research on the distance effect: In Arabic numerals, the physical similarity of the numbers and the Welford function are strongly correlated $(r=.62)$. (As we indicate below, Cohen, 2009a, employed a measure of perceptual similarity based on the seven-line matrix used to create Arabic integers in old digital clocks.) In his experiment, Cohen (2009a) presented participants with single Arabic digits between 1 and 9 and asked them to decide

\footnotetext{
${ }^{1}$ Additional evidence of the automatic processing of integers comes from the size congruency effect ( $\mathrm{SiCE}$ ). In a typical $\mathrm{SiCE}$ experiment, participants are presented with pairs of numbers differing in numerical as well as physical magnitude, and they are required to decide which number is physically larger, while ignoring numerical magnitude. RTs to incongruent trials (i.e., to physically larger but numerically smaller stimuli or physically smaller but numerically larger stimuli) are slower than RTs to congruent trials (Ganor-Stern \& Tzelgov, 2008, Exp. 1; Henik \& Tzelgov, 1982; Tzelgov, Meyer, \& Henik, 1992). However, Cohen (2009a) argued that it is debatable whether the SiCE effect provides an appropriate test of automaticity: The task demands a (physical) magnitude judgment, and this judgment involves the same parietal areas involved in numerical magnitude processing; hence, quantity representations may be activated because of the inherent task demands, rather than by automatic processing of the integers (Cohen, 2009a).
}

whether the number presented was a 5. Participants' average RTs were regressed on the Welford function (i.e., a measure of access to magnitude) and on the physical similarity function. The results showed that both predictors contributed to the regression, but, more importantly, when both functions were included as predictors, only physical similarity was significant. Thus, the findings from Cohen (2009a) pose important problems for the view that numerical magnitude is automatically accessed.

The aim of the present experiments was to shed more light on the underlying processes in a perceptual comparison task with numbers: whether quantity representation (as commonly assumed) or mere perceptual similarity (as argued by Cohen, 2009a) is the basis of the comparison. One potential limitation of Cohen's (2009a) experiment is that his perceptual similarity function was based on logical grounds: He considered both matching and missing features in reference to the seven lines of an 8 matrix in old digital alarm clocks: 123456789 . This measure may work as an initial approach, but it has some obvious limitations. For instance, the numbers 2 and 5 are (in some fonts) nearly the same figure reversed; however, according to Cohen's (2009a) measure, these numbers only show a similarity of 0.75 , which is much smaller than the similarity between 9 and 5 -which is 2 (see Table 1 for the details of Cohen's perceptual similarity function). A purer perceptual similarity function could be obtained by averaging RTs for

Table 1 Calculation of the physical similarity function from Cohen (2009a) when the target integer is a 5 and the distractor integers are 1-4 and 6-9

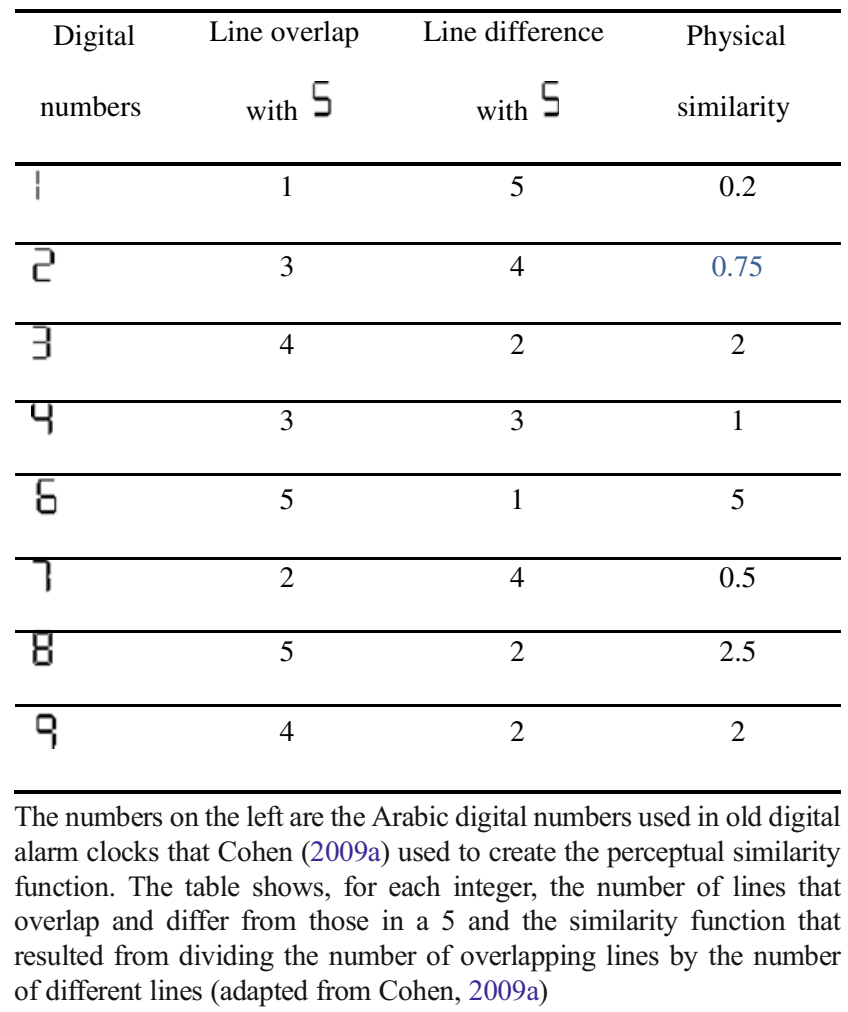


each digit in Cohen's (2009a) perceptual task from participants with no knowledge of Arabic numbers. However, Arabic numerals are so widespread that it is very difficult to obtain such an empirical index. One alternative, which is employed in the present study, is to employ Indian numerals rather than Arabic numerals. Indian numbers constitute a unique set of numerical symbols that are in use in the Arabic world. Presenting Indian numerals to (Spanish) participants without any knowledge of these numbers in a same-different perceptual task provides an empirical measure of the Indian integers' perceptual similarity. This measure, together with the numerical distance function (i.e., the Welford function), was used as a predictor of the RT data of participants who are highly familiar with Indian numerals in two experiments using the same perceptual task as Cohen (2009a).

The present study also takes advantage of the existence of two Indian notations: the Indian numbers ( 1 r $r \varepsilon \circ \neg \vee \wedge 9$ ) used in most Arabic countries, and the Persian variant of Indian numbers ( $\mid r r \nvdash \Delta q \vee \wedge q$ ). (From now onward, we will call these notations the Arabic-Indian and PersianIndian numbers, respectively.) Pilot studies conducted in our lab suggested that a high correlation exists between the distance effect and the perceptual similarity in PersianIndian notation for individuals who don't know this notation, whereas there is no sizeable correlation between these variables in the Arabic-Indian notation; we should note here that, although there is a noticeable similarity between most numbers in the two Indian notations, similarity is computed against the number 5, and this integer is somewhat dissimilar in the two notations ( $\Delta$ in the Persian-Indian and $\odot$ in the Arabic-Indian notation). Consequently, using Persian-Indian and Arabic-Indian numbers allowed us to study the roles of numerical distance and physical similarity when the two variables are highly correlated (Persian-Indian numbers; i.e., a situation similar to that found by Cohen, 2009a, when studying Arabic integers) and when the two variables are not highly correlated (Arabic-Indian numbers), thus providing two different scenarios in which to assess the role of each predictor. In addition, our statistical analyses were more powerful than those employed by Cohen (2009a). Here, we employed a repeated measures regression analysis following Lorch and Myers (1990). This procedure involves computing regression equations for each participant using physical similarity and numerical distance as predictors, and the RTs of participants who are highly familiar with Indian numerals as the predicted variable.

In sum, we conducted two experiments using two different types of Indian notation, using the same task that had been employed by Cohen (2009a) with Arabic integers. In Experiment 1 , we explored the roles of quantity representation versus perceptual similarity in a numerical perceptual task with the Persian-Indian notation. Experiment 2 was parallel to Experiment 1 , except that we employed the Arabic-Indian notation.

\section{Experiment 1}

\section{Method}

Participants A group of 20 participants from Pakistan and 20 native speakers of Spanish (with no knowledge of Indian numbers) participated voluntarily in this experiment. All had normal or corrected-to-normal vision and were naïve regarding the purpose of the study. The Pakistanis were graduates and undergraduates recruited in Valencia (Spain), their ages ranged from 25 to 35 years, and none of them had lived in Spain before 2004. All were native speakers of Urdu, had learned Persian-Indian numbers at school, and were highly familiarized with these numbers - in fact, they used them on a daily basis. The Spanish participants ranged in age from 19 to 29 years, were all undergraduates at the University of Málaga, and had no knowledge of Indian numbers.

Stimuli The trials involved the Persian-Indian integers from 1 to 9: $\backslash\ulcorner r \nvdash \Delta \& \vee \wedge 9$. These numbers are taught at school in Pakistan. Persian-Indian numbers are used mainly in written Arabic materials (e.g., newspapers, books), while Arabic numbers are used in mathematics. Thus, the Pakistani participants included in the present study were familiar with the Arabic as well as with the Persian-Indian symbols.

Procedure The participants were tested individually in a quiet room. They sat in front of a computer monitor located at an approximate distance of $60 \mathrm{~cm}$. The stimuli were presented on a 15 -in color monitor. Presentation of the stimuli and recording of the RTs were controlled by a Windows-based computer using DMDX (Forster \& Forster, 2003). Participants were instructed to decide whether or not the symbol presented was a $\Delta(5$ in Indian script). Number names were not used in the instructions, as the task was intended to be a samedifferent perceptual task. Half of the participants pressed the "M" key if the stimulus presented was a $\Delta$ and the "Z" key if a different stimulus was used, and the response keys were reversed for the other half of the participants. Each experimental trial consisted of the following sequence of events: First, a fixation cross $(+)$ was presented for $500 \mathrm{~ms}$, then the target was presented in Persian-Indian font for 2,500 ms or until the participant's response. The numbers were about $10 \mathrm{~mm}$ high and $7.5 \mathrm{~mm}$ wide. The experiment consisted of 16 practice trials and 544 experimental trials. The target $\Delta$ was presented on half of the practice trials and half of the experimental trials. The remaining integers from 1 to 9 each appeared the same number of times. The stimuli were randomly presented, and a short break was included after every 80 trials. 


\section{Results and discussion}

The error rates were very low for both Pakistanis $(2.25 \%)$ and the Spanish participants (2.52\%). Incorrect responses and RTs less than $250 \mathrm{~ms}$ or greater than $1,000 \mathrm{~ms}$ (less than $1.2 \%$ and $0.6 \%$ of the trials for the Pakistani and the Spanish participants, respectively) were excluded from the latency analysis. Table 2 presents the Welford function values and the mean RTs for Spanish and Pakistani participants with each integer. Overall, Spanish participants $(M=439 \mathrm{~ms}, S D=41)$ were faster than Pakistani participants $(M=488 \mathrm{~ms}, S D=44)$ $[t(38)=4.2, p<.01]$. Additionally, as is usual in the literature (e.g., Sternberg, 1995; see also Cohen, 2009a), responses to the reference target (i.e., "yes" responses) were significantly faster than responses to the different targets (i.e., "no" responses) in both the Pakistani participants $[M=472 \mathrm{~ms}$, $S D=46$, vs. $M=504 \mathrm{~ms}, S D=47 ; \quad t(19)=4.6, p<.001]$ and the Spanish participants $[M=433 \mathrm{~ms}, S D=47$, vs. $M=$ $446 \mathrm{~ms}, S D=36 ; \quad t(19)=3.1, p<.01]$.

To examine the role of perceptual similarity and quantity representation in the perceptual same-different task, regression analyses were conducted using the Pakistanis' RTs for "no" trials as the dependent variable, and the numerical distance (Welford) function and the perceptual similarity function (based on the average RT for Spanish participants at each number) as predictors. In addition, we employed Lorch and Myers's (1990, Method 3) procedure in the analyses. That is, 20 regressions (one for each Pakistani participant) were calculated separately considering the average RTs to each Persian-Indian integer other than 5. Subsequently, the regression coefficients were averaged for each explanatory variable, and the mean coefficient for each explanatory variable was regarded as the overall effect of the variable. Finally, one-sample $t$ tests were used for testing whether this mean effect differed significantly from zero. This procedure is more powerful than the one performed by Cohen (2009a), who regressed the mean RTs of all participants to each integer other than 5 on perceptual similarity and the Welford function.

The correlation between our perceptual index and the Welford function was $r=.65$; this value is very similar to that found by Cohen (2009a) between Arabic numbers and his index of similarity $(r=.62)$. The regression analyses between Pakistanis' RTs and the Welford function showed a significant contribution of quantity representation, slope $=$ $14.89, t(19)=2.28, p=.033$. In addition, a significant relationship was also found when Pakistani participants' RTs were regressed on perceptual similarity (i.e., the average RT for Spanish participants at each number), slope = $0.44, t(19)=4.10, p<.01$ (see Fig. 1 , top). More importantly, when the two factors were entered simultaneously in the regression, using the standardized values of both the predictors and the criterion, the analysis showed no contribution of quantity representation to the regression, slope = $0.002, t(19)=0.04, p=.96$, while the contribution of perceptual similarity was significant, slope $=0.09, t(19)=$ $2.72, p<.02$.

The findings from the present experiment with PersianIndian numerals are clear: A numerical distance effect occurred when this factor was included in a simple regression analysis. However, this seems to be a byproduct of the elevated correlation between quantity representation and perceptual similarity: When both predictors were entered simultaneously in the regression, perceptual similarity was the only factor that significantly predicted RT data in the same-different perceptual task. Although causality can not be concluded from regression analyses, our data suggest that perceptual similarity, rather than quantity representation, guides the response in a task in which participants are asked to decide whether or not the stimuli/numbers presented are perceptually similar. Thus, the present experiment replicates and extends the findings from Cohen (2009a).

In Experiment 2, we took advantage of the existence of another Indian notation: the Arabic-Indian (, r,$\ulcorner, \Sigma, 0,7$,
Table 2 Results from Experiments 1 and 2: Values of the Welford function for each integer and the mean response times (RTs) of Pakistani and Spanish participants in Experiment 1 in a same-different task with Persian-Indian notation, as well as the mean RTs of Jordanian and Spanish participants in a same-different task with ArabicIndian notation in Experiment 2

\begin{tabular}{|c|c|c|c|c|c|}
\hline \multirow[b]{2}{*}{ Integer } & \multirow[b]{2}{*}{ WELFORD } & \multicolumn{2}{|c|}{ Experiment 1: Persian-Indian } & \multicolumn{2}{|c|}{ Experiment 2: Arabic-Indian } \\
\hline & & Pakistani & Spanish & Jordanian & Spanish \\
\hline 1 & 0.10 & 494 & 431 & 454 & 416 \\
\hline 2 & 0.22 & 497 & 432 & 465 & 420 \\
\hline 3 & 0.40 & 508 & 441 & 464 & 416 \\
\hline 4 & 0.70 & 506 & 449 & 461 & 416 \\
\hline 5 & - & 472 & 433 & 433 & 408 \\
\hline 6 & 0.78 & 506 & 461 & 455 & 409 \\
\hline 7 & 0.54 & 504 & 442 & 455 & 412 \\
\hline 8 & 0.43 & 504 & 451 & 467 & 417 \\
\hline 9 & 0.35 & 514 & 462 & 469 & 439 \\
\hline
\end{tabular}



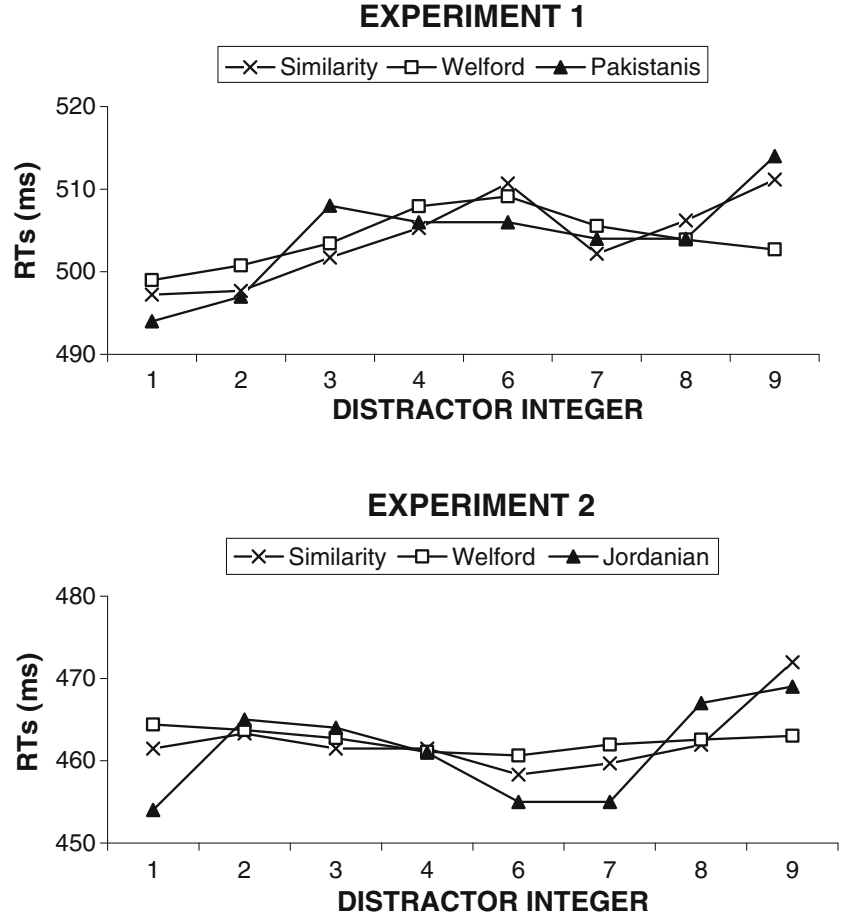

Fig. 1 Results of Experiments 1 and 2. The graph on top shows the mean response times (RTs) for Pakistani participants, the predicted Welford (1960) function, and the predicted similarity function (Exp. 1). When both predictors were included in the regression analysis, only perceptual similarity was a significant predictor of the participants' RTs. The graph on the bottom shows the mean RTs for Jordanian participants, the predicted Welford function, and the predicted similarity function (Exp. 2). Once again, only perceptual similarity was a significant predictor of the participants' RTs

$\vee, \wedge$, and $१$.). This notation is used in most Arabic countries, and a pilot study showed no significant correlation between the perceptual properties of these numbers and their quantity (unlike the parallel correlations when using Arabic integers or Persian-Indian numbers). Thus, this notation would provide a purer test of the "perceptual" view advocated by Cohen (2009a). As we indicated in the introduction, Ganor-Stern and Tzelgov (2008, Exp. 2) did not report numerical distance effects using this notation (nor with Arabic or mixed notations) in a physical same-different task, although evidence of quantity processing was found when presenting mixed pairs with the same numerical value.

\section{Experiment 2}

Method

Participants A group of 30 participants from Jordan and 21 native speakers of Spanish participated in this experiment as volunteers. All had normal or corrected-to-normal vision and were naive regarding the purpose of the study. The
Jordanian participants were undergraduates recruited at the University of Jordan in Amman, and their ages ranged from 19 to 25 years. All were native speakers of Arabic, had learned Arabic-Indian numbers at school, and were highly familiarized with them-using them on a daily basis. The Spanish participants ranged in age from 19 to 32 years, were all graduates and undergraduates recruited at the University of Málaga (Spain), and had no knowledge of Arabic-Indian numbers.

Stimuli The trials involved the Arabic-Indian integers from 1 to $9: 1, r,\ulcorner, \varepsilon, \circ,\ulcorner, \vee, \wedge$, and $`$. These Indian numbers are usually taught at school in most Arabic-speaking countries, including Jordan. They are usually used in written texts, while Arabic numbers are usually employed in calculations. Hence, the Jordanian participants included in the present study were familiar with the Arabic as well as with the Indian symbols.

Procedure This was the same as in Experiment, except that this time participants were instructed to decide whether or not the symbol presented was ${ }^{\circ}$ (5 in Arabic-Indian script).

\section{Results and discussion}

The error rates were low for both the Jordanians (4.06\%) and the Spanish participants $(2.44 \%)$. Incorrect responses and RTs less than $250 \mathrm{~ms}$ or greater than $1,000 \mathrm{~ms}$ (less than $2.3 \%$ and $1.6 \%$ of the trials for the Jordanian and Spanish participants, respectively) were excluded from the latency analysis. In Table 2, we present the mean RTs for the Spanish and Jordanian participants with each ArabicIndian integer. Although the Spanish participants $(M=$ $413 \mathrm{~ms}, S D=67)$ were faster than those with experience with this Indian notation $(M=448 \mathrm{~ms}, S D=73)$, the difference was not significant $[t(49)=1.76, p=.08]$. As in Experiment 1, "yes" responses were significantly faster than "no" responses, for both the Jordanian participants $[M=$ $434 \mathrm{~ms}, S D=71$, vs. $M=462 \mathrm{~ms}, S D=75 ; t(29)=6.9$, $p<.001]$ and the Spanish participants $[M=407 \mathrm{~ms}, S D=69$, vs. $M=418 \mathrm{~ms}, S D=67 ; t(19)=2.14, p<.05]$.

We conducted regression analyses parallel to those in Experiment 1. This time, the correlation between our perceptual index of similarity and the Welford function was negative and considerably smaller $(r=-.39)$ than in the cases of Arabic integers and Persian-Indian numbers. The absence of a sizeable correlation between these two variables offered a good opportunity to explore the role of each variable in predicting participants' responses to our perceptual task. Regression analyses between the Jordanian participants' RTs and the Welford function failed to show effects of quantity representation, slope $=-5.56, t(29)=0.78, p=.44$. 
In contrast, a significant relationship was found when the Jordanian participants' RTs were regressed on perceptual similarity (i.e., the Spanish participants' average RT at each number), slope $=0.45, t(19)=2.71, p<.02$ (see Fig. 1 , bottom). Not surprisingly, the analysis over the standardized values of both predictors, entered simultaneously in the regression equation, showed that the Welford function made no contribution to the equation, slope $=0.004, t(19)=0.23, p=.82$, whereas perceptual similarity was a significant predictor, slope $=0.23$, $t(19)=2.74, p<.02$.

This experiment with Arabic-Indian numerals demonstrates that when there is no significant correlation between perceptual similarity and numerical distance, only perceptual similarity is a reliable predictor of the participants' RTs, thus replicating and extending the findings from Experiment 1.

\section{General discussion}

When we are presented with an integer (e.g., 7) in a task that does not demand access to number quantity, do we automatically access its corresponding semantic representation? Although the accepted view advocates for a "yes" response, recent research has put this claim in question, by showing that, in same-different perceptual tasks, the best predictor of RTs is the physical similarity between numbers, not numerical distance (see Cohen, 2009a). Given the theoretical implications of Cohen's (2009a) experiment, the present study aimed to reexamine this issue by using two different types of Indian notations. The results showed that, regardless of the degree of correlation between the perceptual similarity index and the Welford function (numerical distance), the critical predictor for RTs in the perceptual tasks was the perceptual similarity function, not numerical distance.

Thus, the present findings with Indian numbers are in line with those obtained by Cohen (2009a) with Arabic integers: We failed to find any evidence of automatic access to Indian integers' semantic representations in a perceptual samedifferent task. Importantly, the present experiments are not a mere replication with another script. First, we employed a purer, empirical measure of similarity. The data from (Spanish) participants without any knowledge of the Indian numerals provided a purer index of physical similarity than does a nonempirical index based on an old digital clock. Second, the present regression analyses constitute a more appropriate method for repeated measures designs. Bear in mind that Cohen (2009a) regressed item-by-item reading time differences between number pairs averaged across participants with the Welford function and the similarity function. This analysis is more susceptible to Type I errors and usually leads to an inflation of estimates of the percentages of variance accounted for by the predictors (Lorch \& Myers, 1990). And finally, the use of two Indian notations allowed us to explore the role of quantity representation under two different scenarios: when perceptual similarity and the Welford function are highly correlated (Persian-Indian integers) and when no sizeable correlation exists (Arabic-Indian integers).

Do our findings (together with those of Cohen, 2009a) imply that semantic representations are not necessarily activated upon the presentation of integers? We prefer to remain agnostic on this issue, and argue instead that activation of meaning on the basis of numbers might be too slow to play a significant role in simple perceptual tasks (see Ratinckx, Brysbaert, \& Fias, 2005, Exp. 5, for evidence using masked priming). Furthermore, as Cohen Kadosh and Walsh (2009) indicated, "if the physical shape is more salient than the numerical magnitude, it will mask the effects of the numerical magnitude" (p. 356). That is, the decisions made in same-different perceptual tasks may be based more on physical than on semantic factors (keep in mind that previous evidence with Arabic integers may have been due to the confound between perceptual similarity and numerical distance identified by Cohen, 2009a). The present data are also consistent with the lack of a distance effect reported by Ganor-Stern and Tzelgov (2008) with Arabic-Indian numbers - and note that there is only a small correlation between perceptual similarity and numerical magnitude with this notation. Ganor-Stern and Tzelgov (2008) indicated that there was evidence of semantic processing in that experiment, because RTs were slower for differentnotation pairs with the same numerical values $(2-r)$ than for such pairs with different numerical values $(3-r)$; however, it is arguable that this difference was not semantic in nature because, as was pointed out by Cohen Kadosh, Henik, and Rubinstein (2008), it could have been due to "asemantic transcoding (e.g., due to phonological representation)" (p. 1389).

In sum, the present findings provide a demonstration that participants do not activate quantity representations upon the mere presentation of integers in simple same-different perceptual tasks. Further research should be devoted to examining whether the lack of a distance effect in perceptual tasks is due to the absence of automatic access to the numbers' semantic representations (as was argued by Cohen, 2009a; 2009b) or whether it is due to the (presumably) slow rate of semantic activation from the integers. In this light, one potential option would be to run a samedifferent perceptual task in which physical shape was difficult to process (e.g., via degradation of the stimuli). In this way, there would be more room for semantic activation from the integers to have an effect-assuming that there is automatic access to numerical magnitude. 
Author notes This research was partially supported by Grants PSI2008-06107/PSIC, PSI2009-08889/PSIC, PSI2008-04069/PSIC, and CONSOLIDER-INGENIO2010 CSD2008-00048 from the Spanish government. We thank Cathleen Moore, Elena Salillas and two anonymous reviewers for helpful comments on a previous version of this manuscript.

\section{References}

Cohen, D. J. (2009a). Integers do not automatically activate their quantity representation. Psychonomic Bulletin \& Review, 16, 332-336. doi:10.3758/PBR.16.2.332

Cohen, D. J. (2009b). Numerical representations are neither abstract nor automatic. Behavioral and Brain Sciences, 32, 332-333. doi:10.1017/S0140525X09990549

Cohen Kadosh, R., Henik, A., \& Rubinsten, O. (2008). Are Arabic and verbal numbers processed in different ways? Journal of Experimental Psychology: Learning, Memory, and Cognition, 34, 13771391. doi:10.1037/a0013413

Cohen Kadosh, R., \& Walsh, V. (2009). Numerical representation in the parietal lobes: Abstract or not abstract? Behavioral and Brain Sciences, 32, 313-373. doi:10.1017/S0140525X09990938

Dehaene, S., \& Akhavein, R. (1995). Attention, automaticity, and levels of representation in number processing. Journal of Experimental Psychology: Learning, Memory, and Cognition, 21, 314326. doi:10.1037/0278-7393.21.2.314

Forster, K. I., \& Forster, J. C. (2003). DMDX: A Windows display program with millisecond accuracy. Behavior Research Methods, Instruments, \& Computers, 35, 116-124. doi:10.3758/ BF03195503

Ganor-Stern, D., \& Tzelgov, J. (2008). Across-notation automatic numerical processing. Journal of Experimental Psychology:
Learning, Memory, and Cognition, 34, 430-437. doi:10.1037/ 0278-7393.34.2.430

Henik, A., \& Tzelgov, J. (1982). Is three greater than five: The relation between physical and semantic size in comparison tasks. Memory \& Cognition, 10, 389-395. doi:10.3758/BF03202431

Lorch, R. F., Jr., \& Myers, J. L. (1990). Regression analyses of repeated measures data in cognitive research. Journal of Experimental Psychology: Learning, Memory, and Cognition, 16, 149157. doi:10.1037/0278-7393.16.1.149

Moyer, R. S., \& Landauer, T. K. (1967). Time required for judgments of numerical inequality. Nature, 215, 1519-1520. doi:10.1038/ $2151519 \mathrm{a} 0$

Ratinckx, E., Brysbaert, M., \& Fias, W. (2005). Naming two-digit Arabic numerals: Evidence from masked priming studies. Journal of Experimental Psychology: Human Perception and Performance, 31, 1150-1163. doi:10.1037/0096-1523.31.5.1150

Sternberg, S. (1995). Inferring mental operations from reaction-time data: How we compare objects. In D. Scarborough \& S. Sternberg (Eds.), Methods, models, and conceptual issues (pp. 365-454). Cambridge, MA: MIT Press.

Tzelgov, J. (1997). Specifying the relations between automaticity and consciousness: A theoretical note. Consciousness and Cognition, 6, 441-451. doi:10.1006/ccog.1997.0303

Tzelgov, J., \& Ganor-Stern, D. (2005). Automaticity in processing ordinal information. In J. I. D. Campbell (Ed.), Handbook of mathematical cognition (pp. 55-67). New York, NY: Psychology Press.

Tzelgov, J., Meyer, J., \& Henik, A. (1992). Automatic and intentional processing of numerical information. Journal of Experimental Psychology: Learning, Memory, and Cognition, 18, 166-179. doi:10.1037/0278-7393.18.1.166

Welford, A. T. (1960). The measurement of sensory-motor performance: Survey and reappraisal of twelve years' progress. Ergonomics, 3, 189-230. 